\title{
The Application of Mind and Breath in Erhu Performance
}

\author{
Xi Shuting \\ Zaozhuang University, Shandong, China
}

Keywords: erhu performance; idea; breath; use

Abstract: Erhu is a traditional Chinese musical instrument with beautiful timbre and rich music expressive force. The use of ideas and breath in erhu performance is directly related to the performance quality, and the proper use of ideas and breath can also show the deep understanding of erhu players on erhu performance works. Therefore, the use of breath plays a crucial role in the performance of erhu performers. The breath and idea of the performers dominate the progress of the music. This paper first briefly introduces the concept of idea and breath in erhu performance, then expounds the importance of idea and breath in erhu performance, and finally studies the application of idea and breath in erhu performance, in order to improve the level of breath control and complete performance of erhu works.

\section{Introduction}

Reasonable use and control of the scent and thoughts in the erhu performance is actually able to dominate the main theme of a work. When we play the erhu, we can clearly feel that we can use our "mouth", "ventilation", "cohesion", that is, breath, we must use our ideas to achieve "control". It plays a vital role in the perfection of the second creation of the erhu works. Only in the erhu performance, the use of the breath and the profound understanding of the performance of the performance, can really use the idea to control the atmosphere of the performance, so that we can guarantee the high quality and perfection of our erhu works ${ }^{[1]}$.

\section{The concept of mind and breath in erhu performance}

\subsection{Breath in the erhu performance}

The motive force for the sound of the music is the breath. The breath means that different breathing techniques are used in different marks of the erhu works to fully express the inner meaning of the music. The slow-sounding and solemn scores require the performers to ensure a smooth and even application of the sound during the performance, fully coordinate the passages in the scores, and use our own musical literacy and understanding of the musical works. In the section of the good wood section music, there are no obvious marked breaths, and the coherence of the upper and lower music and the mastery of the breath are fully completed. The air outlet in the movement is like the punctuation mark in an article, so when you encounter this part in the erhu 
performance, you should use a steady mouth and a suction to use the mouth, do not have ups and downs, keep coherence, not tight. Slowly maintaining the breath will make the stability of the bow a coherent, and it will also ensure the perfect sound and performance of the erhu works. The relevant part of the study on the use of breath in erhu performance is shown in Table 1.

Table 1 Factors affecting the use of erhu performers

\begin{tabular}{|l|l|}
\hline Influential factors & Player request \\
\hline Breathe & Smooth breath \\
\hline Mentality & Concentrated \\
\hline Posture & Correct posture \\
\hline
\end{tabular}

\subsection{Ideas in erhu performance}

Through the performance of the erhu works, it achieves the shocking effect, showing the image of the music works and the profound connotation of the music works. When we play erhu works, if we want to express the inner meaning and external image of the music works, then we should pay more attention to the harmony with the connotation image of the works in the use of breath, and achieve mutual perfection. For example, A Bing's famous song "Two Springs Reflecting the Moon", the whole work is showing an artistic conception. Therefore, through the performance of erhu music works, it can achieve an artistic conception and a spiritual experience that spans time and space. It is this kind of emotion that triggers the artistic "ideas" of art. When playing music works, it uses the mind to control the breath and the bow. Techniques, in order to achieve the "reality of heaven and man" performance ${ }^{[2]}$. Figure 1 is a diagram of the process of thinking and thinking of instrumentalists.

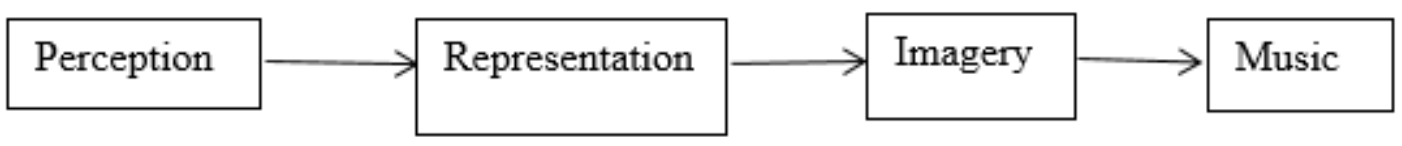

Figure 1 Instrumentalist thinking process

\section{The importance of mind and breath in erhu performance}

Erhu performer before the formal performance, often need to calm his heart, to grasp the essence of the erhu works, let oneself quickly into the state of play in the play the erhu in the process of the traditional ethnic Musical Instruments, players tend to pursue the busines, but quiet performance places tend to bring players nerves, this requires players to use their own will and mind, to reasonable control and use of breath, and let their emotions quickly stabilized, and works of perfection the erhu performer in the play in the process of actual performance, often based on the change of status, Adjust and control the breath to make yourself better and ensure the performance effect. At the end of the performance, the performer also needs to go through the process of drawing in and exhaling, just like tai chi, a set of kung fu will be relaxed immediately after the end. This is because in the performance of erhu music, we devote ourselves wholeheartedly to it and display every phrase perfectly. This kind of whole-hearted concentration is not disturbed by external things, and it is a kind of concentration that is always integrated into music works ${ }^{[3]}$.

In our highly integrated works, of course, we will constantly change our playing emotions along with the ups and downs of music works, and our mind can always keep a tight and loose state when we play. Naturally keeping relaxed is the most important state of mind in erhu performance. Once the stiffness of fingers occurs, it will affect the performance effect and affect the bow movement and finger movement of the left and right hands. The best way to deal with this is to use breath to 
relax. When we are fully integrated with the music works and achieve the artistic conception of "unity of man and man", the tension will be naturally eliminated and our attention will no longer be focused on our right and left hands. In the process of performance, the artistic conception can control the breath. Only when the connotation of erhu music works is fully understood and integrated into it, can the perfect performance effect be displayed.

\section{Breathing and mentality in erhu performance}

\subsection{Breathing while playing the erhu}

The players have three breathing states, pre-adjustment state, in-performance state and natural state. Pre-performance state adjustment is often said to be heavy, this adjustment process is very important, can help the player into the state. The state of performance is mostly based on the natural state. Through long-term training and practice, the breathing power formed by interaction and adaptation with performance has two characteristics of rules and no rules. Because the breath is constantly changing with the music of the performance, it is affected by the content, rhythm, skill, strength and other factors. Compared with the natural state, it is irregular. But the regular characteristic is that the general erhu performance must follow the characteristics of drawing the bow to inhale and pushing the bow to exhale.

\section{2 mentality while playing the erhu}

To do anything well requires concentration, so does the performance of the erhu, which requires eliminating distractions and entering the playing scene through imagination. In the process of performance, players can not only express their emotions through facial, language and body language, but also show their changes in mentality through changes in breath. When it comes to the passionate section, the state of mind will also become more intense, and the flow of breath will increase, while when the section is deep and beautiful, the performance will slow down, the state of mind is also relatively calm, and the breath is consistent with the state of mind, which reflects the correlation between breath and the change of state of mind ${ }^{[4]}$.

\section{The use of breath and mind in erhu performance}

Breath and mind will have a direct impact on the level of erhu performance. Only by breathing with the music works can the breath and mind be integrated in the music, improve the expressive power of the music, and also show the player's understanding and comprehension of the work. Table 2 is about the use of breath and ideas in the whole process of erhu performance. Players should accumulate breath and ideas in a large number of performances and training, and play the best level in the stage performance. On the one hand, erhu performers should pay attention to the use of breath combination movements in performance, that is, to combine different breathing methods. Before the exercise, you need to adjust the breath effectively, first take a deep breath with your nose, and let the gas feel first. In order to make the sound of the erhu play more full and beautiful, you need to follow the principle of inhaling and pushing the bow. In the process of playing the whole erhu works, it is necessary to divide the music based on emotions and reasonably carry out the breath combination action. 
Table 2 The use of breath and mind in the whole process of erhu performance

\begin{tabular}{|ll|}
\hline \multicolumn{2}{|c|}{ The use of breath and mind in the whole process of erhu performance } \\
\hline$\bullet$ & Marking and breath on the spectrum \\
\hline$\bullet$ & The image and mind and breath of the work \\
\hline & Playing state and mind and breath \\
\hline
\end{tabular}

On the other hand, the training of breath and mind in erhu performance can draw on the spirit and method of breath in Qigong practice. Erhu performance is a form of artistic practice that combines the physiological and psychological laws of the human body. This qigong spirit is common and requires the adjustment of meaning, body and mind. The dancers are required to be able to eliminate distractions and quickly enter the realm of performance by comprehending musical works. The tempering requires the player to regulate and adjust his or her performance posture. After sitting, the waist should be straight and straighten to find a suitable playing sensation. The adjustment of the rate requires the player to control his or her own mind, so that the breathing can be adjusted, so that his breath can quickly sink, and the imagination can be opened into the playing world. Commonly used breath adjustment methods are abdominal, chest breathing and natural breathing. Erhu players can choose the appropriate breath adjustment method according to the work and their own needs, and use the reasonable use of ideas in the performance to improve the quality of the performance ${ }^{[5]}$.

\section{Conclusions}

The performance process of erhu is the second creation process of erhu works. Skilled use thoughts to control breath is very important in the erhu, good to erhu play effect, the performer must have skillful techniques and the music master, combined with the use of mind control breath, really play breath synchronization with the music "breathing", this play will present a perfect effect. Erhu players must pay attention to the fact that in the process of erhu playing, the playing breath and the music "breath" are synchronized to achieve the state of "emotion, skill and heart". Only in erhu performance is good at using the idea to control the breath can truly achieve the "hu unity" realm.

\section{References}

[1] Zhang Jinfeng. Analysis of the Application of Breath in Erhu Performance [J]. Music Time and Space, 2014(8): 123-123.

[2] Ma Lina. The Ideas and Breath in the Performance of Pelicans[J]. Northern Music, 2013(10): 139-139.

[3] Wang Xiaohong. On the Sound Processing of Erhu[J]. Northern Music, 2013(10): 135-136.

[4] Feng Yuting. On the use of breath in the performance of pipa and the emotional expression of second creation [D]. Xi'an Conservatory of Music, 2017.

[5] Lei Yuxi. Talking about the timbre of Erhu [J]. User World, 2014(11): 128-128. 